\title{
PERTURBED MAP RISK MODELS WITH DIVIDEND BARRIER STRATEGIES
}

\author{
ERIC C. K. CHEUNG* AND \\ DAVID LANDRIAULT, ${ }^{* * *}$ University of Waterloo
}

\begin{abstract}
In the context of a dividend barrier strategy (see, e.g. Lin, Willmot and Drekic (2003)) we analyze the moments of the discounted dividend payments and the expected discounted penalty function for surplus processes with claims arriving according to a Markovian arrival process (MAP). We show that a relationship similar to the dividend-penalty identity of Gerber, Lin and Yang (2006) can be established for the class of perturbed MAP surplus processes, extending in the process some results of $\mathrm{Li}$ and $\mathrm{Lu}$ (2008) for the Markovmodulated risk model. Also, we revisit the same ruin-related quantities in an identical MAP risk model with the only exception that the barrier level effective at time $t$ depends on the state of the underlying environment at this time. Similar relationships are investigated and derived. Numerical examples are also considered.
\end{abstract}

Keywords: Markovian arrival; perturbed process; barrier strategy; Gerber-Shiu function; discounted dividend payment

2000 Mathematics Subject Classification: Primary 60J75

Secondary $60 \mathrm{~J} 25 ; 60 \mathrm{~J} 60$

\section{Introduction}

In this paper we consider surplus processes for which the claim number process $\{N(t), t \geq 0\}$ is a Markovian arrival process (MAP). A MAP with representation $\operatorname{MAP}(\boldsymbol{\alpha}, \boldsymbol{D}, \boldsymbol{T})$ of order $m$ is a two-dimensional Markov process on the state space $\mathbb{N} \times\{1, \ldots, m\}$ for which the first dimension reflects the evolution of the total number of claims over time and the second dimension refers to the evolution of an underlying homogeneous continuous-time Markov chain (CTMC) $\underline{J}=\{J(t), t \geq 0\}$ with finite state space $E=\{1, \ldots, m\}$. For such a process, we subdivide the transitions of the MAP into the following two types.

Type 1. Transitions of the CTMC $\underline{J}$ from state $i$ to state $j(j \neq i)$ without an accompanying claim.

Type 2. Transitions of the CTMC $\underline{J}$ from state $i$ to state $j$ (with possibly $i=j$ ) with an accompanying claim.

Transitions of type 1 are governed by the matrix $\boldsymbol{D}$ for which its $(i, j)$ th element $D_{i j}$ $\left(D_{i j} \geq 0\right)$ corresponds to the instantaneous rate of transition from state $i$ to state $j \neq i$ in $E$ without an accompanying claim. Type- 2 transitions are governed by the matrix $\boldsymbol{T}$ for which its $(i, j)$ th element $T_{i j}\left(T_{i j} \geq 0\right)$ corresponds to the instantaneous rate of transition from state $i$ to state $j$ in $E$ with an accompanying claim. The diagonal elements of $\boldsymbol{D}$ are assumed to be

Received 28 February 2008; revision received 20 March 2009.

* Postal address: Department of Statistics and Actuarial Science, University of Waterloo, 200 University Avenue West,

Waterloo, Ontario, N2L 3G1, Canada.

** Email address: dlandria@math.uwaterloo.ca 
negative and such that the sums of the elements on each row of the generator $\boldsymbol{D}+\boldsymbol{T}$ are all 0 . We denote by $\boldsymbol{\alpha}$ the initial (i.e. at time 0 ) probability vector of the underlying CTMC. For a detailed treatment of MAPs, we refer the reader to, e.g. Latouche and Ramaswami (1999) and Neuts (1989).

For type- 2 transitions, we assume that the distribution of the accompanying claim size can depend on the state of the CTMC $\underline{J}$ immediately before and after the transition. Thus, for a type-2 transition of the CTMC from state $i$ to state $j$, the accompanying claim size is

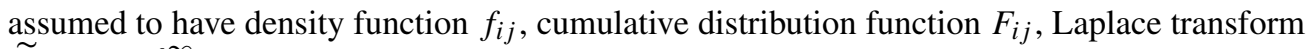
$\widetilde{f}_{i j}(s)=\int_{0}^{\infty} \mathrm{e}^{-s x} f_{i j}(x) \mathrm{d} x$, and finite mean $\mu_{i j}$. In this paper we denote by $X_{n}$ the size of the $n$th claim. From the above construction, it is immediate that the random variables (RVs) $\left\{X_{n}\right\}_{n \geq 1}$ are not independent (in general); conditional on $\underline{J}$, the claim size RVs $\left\{X_{n}\right\}_{n \geq 1}$ are independent.

Remark 1. Both the Markov-modulated risk model (see, e.g. Asmussen (1989), Lu and Tsai (2007), and the references therein) and Albrecher and Boxma's (2005) semi-Markovian risk model are special cases of a risk model with Markovian claim arrivals for which the matrices $\boldsymbol{T}$ and $\boldsymbol{D}$, respectively, in the MAP representation are assumed to be diagonal.

To account for small fluctuations in the surplus level, we assume that the surplus process is perturbed by a Wiener process with mean 0 and volatility $\sigma_{i}\left(\sigma_{i}>0\right)$ whenever the CTMC $J$ is in state $i$. It is also assumed that the insurer collects premiums at a rate $c_{i}$ whenever the environment process $\underline{J}$ is in state $i$. Under the above assumptions, the surplus process $\underline{U}=\{U(t), t \geq 0\}$ is defined as

$$
U(t)=u+\int_{0}^{t} c_{J(s)} \mathrm{d} s-\sum_{n=1}^{N(t)} X_{n}+\int_{0}^{t} \sigma_{J(s)} \mathrm{d} W(s),
$$

where $u$ is the insurer's nonnegative initial surplus and $\{W(t), t \geq 0\}$ is a standard Wiener process, independent of the claim sizes $\left\{X_{n}\right\}_{n \geq 1}$ and the claim number process $\{N(t), t \geq 0\}$. For the surplus process $\underline{U}$, the time to ruin $T$ is defined as $T=\inf \{t \geq 0: U(t) \leq 0\}$ with $T=\infty$ if ruin does not occur (i.e. $U(t)>0$ for all $t \geq 0$ ).

In the presence of a dividend barrier at level $b>0$, the barrier-modified version of the surplus process $\underline{U}$ is denoted by $\left\{U_{b}(t), t \geq 0\right\}$. For such a dividend strategy, it is assumed that whenever the (modified) surplus reaches level $b$, the overflow is paid as dividends; otherwise, no dividend is paid (see, e.g. Lin et al. (2003) and Gerber and Shiu (2004a)). To provide a formal definition of $\left\{U_{b}(t), t \geq 0\right\}$, we define the running maximum of the surplus process $\underline{U}$ at time $t$ by

$$
M(t)=\max _{0 \leq y \leq t} U(y) .
$$

It follows that the total of the (nondiscounted) dividend payments by time $t$ is

$$
D(t)=\max ((M(t)-b), 0) .
$$

This leads to the following definition of the barrier-modified surplus process $\left\{U_{b}(t), t \geq 0\right\}$ :

$$
U_{b}(t)=U(t)-D(t)
$$

Pertaining to the surplus process (1) is the time to ruin $T_{b}=\inf \left\{t \geq 0: U_{b}(t) \leq 0\right\}$, which is finite almost surely (a.s.). 
Remark 2. In the ruin theory literature, it is common to express ruin-related quantities defined in a barrier-type environment in terms of the same ruin-related quantities defined in an identical but barrier-free environment (see, e.g. Lin et al. (2003), Li and Lu (2008), and the references therein). For surplus processes defined in a barrier-free environment, a condition known as the positive security loading condition is generally assumed to be satisfied (to avoid some technicalities that arise otherwise). In our setup, this positive security loading condition is

$$
\sum_{i=1}^{m} \pi_{i} \sum_{j=1}^{m} T_{i j} \mu_{i j}<\sum_{i=1}^{m} \pi_{i} c_{i}
$$

where the $\pi_{i}$ s are the stationary probabilities of the CTMC $\underline{J}$, which are the solutions of the following system of linear equations:

$$
\sum_{j=1, j \neq i}^{m} \pi_{j}\left(D_{j i}+T_{j i}\right)=\pi_{i} \sum_{j=1, j \neq i}^{m}\left(D_{i j}+T_{i j}\right), \quad i \in E,
$$

and

$$
\sum_{j=1}^{m} \pi_{j}=1
$$

We point out that the left- and right-hand sides of (2) correspond to the long-run claim expense and the premium income, respectively, per unit time.

Applications of MAPs in a ruin-theoretical context can be found in, e.g. Ahn et al. (2007), Badescu et al. (2005), (2007), and the references therein. Owing to the elegant interplay between formal algebraic manipulations of mathematical expressions and their probabilistic interpretations, matrix-analytic methods (MAMs) have been instrumental to the analysis of risk models with Markovian claim arrival processes. From the existing connection between fluid flows and surplus processes (see, e.g. Asmussen (1995)), the analysis of these risk models has benefited from the development of MAMs in the fluid flow literature (see, e.g. Ahn et al. (2007)). In general, the fluid flow type analysis of MAP risk models is more probabilist in nature and relies heavily on the knowledge of the Laplace transform of various first passage times (see, e.g. Ahn and Ramaswami (2006) and Ramaswami (2006)). However, a drawback of these fluid flow MAMs for the purpose of ruin theory applications is their limitation to claim size distributions that are phase type, excluding heavy-tail claim size distributions (among others) from the analysis. In this paper we propose to rely on a purely analytic approach to analyze MAP risk models. A variety of theoretical results are derived for general claim size distributions $f_{i j}(i, j=1, \ldots, m)$. Remarks on their numerical implementation and some possible limitations will be made as appropriate.

The rest of the paper is structured as follows. In Sections 2 and 3 we analyze the moments of the discounted dividend payments and the expected discounted penalty function for the surplus process $\left\{U_{b}(t), t \geq 0\right\}$, respectively. In Section 3 we also show that a dividend-penalty identity type relationship holds for the class of perturbed MAP surplus processes, extending in the process some results of $\mathrm{Li}$ and $\mathrm{Lu}$ (2008) for the Markov-modulated risk model. In Section 4, all these ruin-related quantities are revisited in an identical MAP risk model subject to a dividend barrier strategy for which the barrier level effective at a given time $t$ depends on the state of the CTMC $\underline{J}$ at that time. A numerical illustration follows. 


\section{Discounted dividend payments}

In this section we analyze the moments of

$$
D_{u, b}=\int_{0}^{T_{b}} \mathrm{e}^{-\delta y} \mathrm{~d} D(y),
$$

representing the total discounted dividend payments before ruin for the surplus process (1), where $\delta(\delta \geq 0)$ is the force of interest.

\subsection{Expected discounted dividend payments}

Let $V_{i}(u ; b)$ be the expected discounted dividend payments before ruin, assuming that the initial state of the CTMC $\underline{J}$ is $i(i \in E)$, i.e.

$$
V_{i}(u ; b)=\mathrm{E}\left[D_{u, b} \mid J(0)=i\right] .
$$

By an application of Itô's formula for jump diffusion processes together with the fact that the drift component of the resulting stochastic differential equation shall be 0 (due to the presence of a dividend barrier), we readily obtain the following system of integro-differential equations for $V_{i}(u ; b)(i \in E)$ :

$$
\begin{aligned}
& \frac{\sigma_{i}^{2}}{2} V_{i}^{\prime \prime}(u ; b)+c_{i} V_{i}^{\prime}(u ; b)-\delta V_{i}(u ; b)+\sum_{k=1}^{m} D_{i k} V_{k}(u ; b) \\
& +\sum_{k=1}^{m} T_{i k} \int_{0}^{u} V_{k}(u-x ; b) f_{i k}(x) \mathrm{d} x=0, \quad 0 \leq u \leq b .
\end{aligned}
$$

Boundary conditions associated to the system of integro-differential equations (3) for $V_{i}(u ; b)$ are

$$
V_{i}(0 ; b)=0
$$

and

$$
V_{i}^{\prime}(b ; b)=1
$$

for $i \in E$. Equation (4) is immediate given that ruin occurs immediately a.s. when the initial surplus is 0 (due to the diffusion component). Boundary condition (5) is a special case of (21), below, at $n=1$. Thus, the reader is referred to the proof of (21) in Section 2.2 instead.

To determine the form of the solution for $V_{i}(u ; b)$, we consider the system of integrodifferential equations

$$
\begin{aligned}
& \frac{\sigma_{i}^{2}}{2} v_{i}^{\prime \prime}(u)+c_{i} v_{i}^{\prime}(u)-\delta v_{i}(u)+\sum_{k=1}^{m} D_{i k} v_{k}(u) \\
& +\sum_{k=1}^{m} T_{i k} \int_{0}^{u} v_{k}(u-x) f_{i k}(x) \mathrm{d} x=0, \quad u \geq 0 \text { and } i \in E .
\end{aligned}
$$

From the form of the integro-differential equation (6), it is clear that the initial conditions $\left(v_{1}(0), \ldots, v_{m}(0)\right)$ and $\left(v_{1}^{\prime}(0), \ldots, v_{m}^{\prime}(0)\right)$ uniquely determine the solutions $\left(v_{1}(u), \ldots\right.$, $\left.v_{m}(u)\right)$ of system (6). Thus, for a given $j \in E$, let $\boldsymbol{v}_{\cdot, j}^{A}(u)=\left(v_{1, j}^{A}(u), \ldots, v_{m, j}^{A}(u)\right)$ and $\boldsymbol{v}_{\cdot, j}^{B}(u)=\left(v_{1, j}^{B}(u), \ldots, v_{m, j}^{B}(u)\right)$ be the particular solutions of (6) with initial conditions 
$\boldsymbol{v}_{\cdot, j}^{A}(0)=\boldsymbol{e}_{j},\left(\boldsymbol{v}_{\cdot, j}^{A}\right)^{\prime}(0)=\mathbf{0}$ and $\boldsymbol{v}_{\cdot, j}^{B}(0)=\mathbf{0},\left(\boldsymbol{v}_{\cdot, j}^{B}\right)^{\prime}(0)=\boldsymbol{e}_{j}$, respectively, where $\boldsymbol{e}_{j}$ is an $m$-dimensional row vector with the only nonnull entry of 1 at the $j$ th position and $\mathbf{0}$ is an $m$-dimensional row vector of 0 s. Clearly, the solutions $\left\{\boldsymbol{v}_{\cdot, j}^{A}(u)\right\}_{j=1}^{m}$ and $\left\{\boldsymbol{v}_{\cdot, j}^{B}(u)\right\}_{j=1}^{m}$ are linearly independent. In this paper we adopt the convention that the derivative of a matrix/vector holds for an identical size matrix/vector whose elements are entrywise differentiated. Thus, the general solution $\left(v_{1}(u), \ldots, v_{m}(u)\right)$ of $(6)$ can be expressed as

$$
v_{i}(u)=\sum_{j=1}^{m} v_{j}(0) v_{i, j}^{A}(u)+\sum_{j=1}^{m} v_{j}^{\prime}(0) v_{i, j}^{B}(u), \quad u \geq 0 .
$$

In particular, for the expected discounted dividend payments, we have

$$
V_{i}(u ; b)=\sum_{j=1}^{m} V_{j}(0 ; b) v_{i, j}^{A}(u)+\sum_{j=1}^{m} V_{j}^{\prime}(0 ; b) v_{i, j}^{B}(u), \quad 0 \leq u \leq b .
$$

Incorporating boundary condition (4) into (7) leads to

$$
V_{i}(u ; b)=\sum_{j=1}^{m} V_{j}^{\prime}(0 ; b) v_{i, j}^{B}(u)
$$

From (8), boundary condition (5) can be rewritten as

$$
V_{i}^{\prime}(b ; b)=\sum_{j=1}^{m} V_{j}^{\prime}(0 ; b)\left(v_{i, j}^{B}\right)^{\prime}(b)=1, \quad i \in E .
$$

Let $\boldsymbol{v}^{B}(u)$ be a square matrix with element $v_{i, j}^{B}(u)$ at the $(i, j)$ th position, and let $\boldsymbol{V}(u ; b)=$ $\left(V_{1}(u ; b), \ldots, V_{m}(u ; b)\right)^{\top}$. In matrix form, (9) can be expressed as

$$
\boldsymbol{V}^{\prime}(0 ; b)=\left[\left(\boldsymbol{v}^{B}\right)^{\prime}(b)\right]^{-1} \mathbf{1},
$$

where 1 is an $m$-dimensional column vector of $1 \mathrm{~s}$. Combining (8) and (10), the expected discounted dividend payments admits the representation

$$
\boldsymbol{V}(u ; b)=\boldsymbol{v}^{B}(u)\left[\left(\boldsymbol{v}^{B}\right)^{\prime}(b)\right]^{-1} \mathbf{1}, \quad 0 \leq u \leq b .
$$

Equation (11) gives the first moment of $D_{u, b}$ conditional on the initial state $J(0)$ of the underlying CTMC. Premultiplying both sides of (11) by the initial probability vector $\alpha$ yields the first unconditional moment of $D_{u, b}$. In what follows, results are given in their conditional form only. It is understood that their unconditional counterparts can be obtained by premultiplying the conditional representation of a given ruin-related quantity by $\boldsymbol{\alpha}$.

From representation (11) of $\boldsymbol{V}(u ; b)$ we devote the rest of this subsection to the particular solutions $\left\{\boldsymbol{v}_{\cdot, j}^{B}(u)\right\}_{j=1}^{m}$. Let $\widetilde{v}_{i, j}^{B}(s)=\int_{0}^{\infty} \mathrm{e}^{-s u} v_{i, j}^{B}(u) \mathrm{d} u$ for $i, j \in E$. Taking Laplace transforms on both sides of (6) by first replacing $v_{i}(u)$ by $v_{i, j}^{B}(u)$, we obtain

$$
\left(\frac{\sigma_{i}^{2}}{2} s^{2}+c_{i} s-\delta\right) \widetilde{v}_{i, j}^{B}(s)+\sum_{k=1}^{m} D_{i k} \widetilde{v}_{k, j}^{B}(s)+\sum_{k=1}^{m} T_{i k} \widetilde{v}_{k, j}^{B}(s) \widetilde{f}_{i k}(s)=\frac{\sigma_{i}^{2}}{2}\left(v_{i, j}^{B}\right)^{\prime}(0) .
$$


Letting $\widetilde{\boldsymbol{v}}^{B}(s)=\left(\widetilde{v}_{i, j}^{B}(s)\right)_{i, j=1}^{m}$, the system of equations (12) can be expressed in a matrix form as

$$
\boldsymbol{A}(s) \widetilde{\boldsymbol{v}}^{B}(s)=\operatorname{diag}\left(\frac{\sigma_{1}^{2}}{2}, \ldots, \frac{\sigma_{m}^{2}}{2}\right),
$$

where $\boldsymbol{A}(s)=\boldsymbol{P}(s)+\boldsymbol{D}+\boldsymbol{T}(s)$,

$$
\boldsymbol{P}(s)=\operatorname{diag}\left(\frac{\sigma_{1}^{2}}{2} s^{2}+c_{1} s-\delta, \ldots, \frac{\sigma_{m}^{2}}{2} s^{2}+c_{m} s-\delta\right),
$$

and $\boldsymbol{T}(s)$ is a square matrix (of size $m$ ) with element $T_{i k} \widetilde{f}_{i k}(s)$ in position $(i, k)$. Letting adj $\boldsymbol{A}(s)$ be the adjoint matrix of $\boldsymbol{A}(s)$, we conclude that

$$
\widetilde{v}_{i, j}^{B}(s)=\frac{\sigma_{j}^{2}}{2} \frac{[\operatorname{adj} \boldsymbol{A}(s)]_{i, j}}{\operatorname{det} \boldsymbol{A}(s)} .
$$

A Laplace transform inversion of (13) leads to the identification of $v_{i, j}^{B}(u)(i, j \in E)$. Two cases will be discussed separately here.

(a) When all the claim size densities $f_{i j}(i, j \in E)$ have a rational Laplace transform (see, e.g. Dufresne (2001)), the Laplace transform (13) can be inverted analytically. Indeed, let

$$
\tilde{f}_{i j}(s)=\frac{p_{i j}(s)}{q_{i j}(s)}, \quad i, j \in E,
$$

where $p_{i j}(s)$ is a polynomial of degree less than $r_{i j}$ and $q_{i j}(s)$ is a polynomial of degree $r_{i j}$ with $p_{i j}(0) / q_{i j}(0)=1$. It is immediate that the characteristic equation $\operatorname{det} \boldsymbol{A}(s)=0$ has $2 m+r$ solutions, say $\left\{\rho_{i}\right\}_{i=1}^{2 m+r}$, with $r=\sum_{i=1}^{m} \sum_{j=1}^{m} r_{i j}$. For simplicity, we assume that the solutions $\left\{\rho_{i}\right\}_{i=1}^{2 m+r}$ are distinct.

Letting $q(s)=\prod_{i=1}^{m} \prod_{j=1}^{m} q_{i j}(s),(13)$ becomes

$$
\widetilde{v}_{i, j}^{B}(s)=\frac{\sigma_{j}^{2}}{2} \frac{\gamma_{i j}(s)}{\gamma(s)},
$$

where $\gamma_{i j}(s)=q(s)[\operatorname{adj} \boldsymbol{A}(s)]_{i, j}$ and $\gamma(s)=q(s) \operatorname{det} \boldsymbol{A}(s)$ are polynomials of degrees less than $2 m+r$ and $2 m+r$, respectively. For an arbitrary $\kappa$ with $\kappa \neq \rho_{i}$ for $i=$ $1, \ldots, 2 m+r$, the use of Lagrange's interpolating polynomial allows us to rewrite (14) as

$$
\widetilde{v}_{i, j}^{B}(s)=\frac{\sigma_{j}^{2}}{2 \gamma(\kappa)} \sum_{l=1}^{2 m+r} \gamma_{i j}\left(\rho_{l}\right) \beta_{l}(\kappa) \frac{\rho_{l}-\kappa}{\rho_{l}-s},
$$

where $\beta_{l}(s)=\prod_{k=1, k \neq l}^{2 m+r}\left(\left(\rho_{k}-s\right) /\left(\rho_{k}-\rho_{l}\right)\right)$. Inverting (15) yields

$$
v_{i, j}^{B}(u)=\frac{\sigma_{j}^{2}}{2 \gamma(\kappa)} \sum_{l=1}^{2 m+r} \gamma_{i j}\left(\rho_{l}\right) \beta_{l}(\kappa)\left(\kappa-\rho_{l}\right) \mathrm{e}^{\rho_{l} u}, \quad u \geq 0 .
$$

Combining (11) and (16), a closed-form expression for $\boldsymbol{V}(u ; b)$ can be readily found.

(b) When one or more claim size densities $f_{i j}$ do not have a rational Laplace transform, we will most likely have to rely on numerical Laplace transform inversion methods (see, e.g. Abate et al. (2000)) to invert (13) in order to evaluate $\boldsymbol{V}(u ; b)$ via (11). 
The following example considers the two cases discussed above.

Example 1. We consider a risk model for which claims arrive according to a MAP with

$$
\boldsymbol{D}=\left[\begin{array}{cc}
-0.045 & 0.005 \\
0.02 & -0.2
\end{array}\right] \text { and } \boldsymbol{T}=\left[\begin{array}{cc}
0.03 & 0.01 \\
0.04 & 0.14
\end{array}\right]
$$

We assume that premiums are collected at a rate of three per unit time in both states of the CTMC $\underline{J}$. The volatility coefficients are $\sigma_{i}=0.1 i$ for $i=1,2$.

Scenario 1 . We assume that the claim size densities $f_{i j}$ are exponentially distributed with mean $1 / \beta_{i j}$ with $\beta_{11}=0.5, \beta_{12}=0.1, \beta_{21}=0.2$, and $\beta_{22}=0.05$.

Scenario 2. We assume that the claim size densities $f_{i 1}(i=1,2)$ are as stated in scenario 1 . However, the densities $f_{12}$ and $f_{22}$ are substituted by heavy-tail distributions with the following Pareto densities:

$$
f_{i 2}(x)=\beta_{i 2} \theta_{i 2} \frac{\left(\theta_{i 2}-1\right)^{\theta_{i 2}}}{\left(\theta_{i 2}-1+\beta_{i 2} x\right)^{\theta_{i 2}+1}}, \quad x>0,
$$

for $i=1,2$ with $\theta_{12}=5$ and $\theta_{22}=3$.

Note that the densities $f_{i 2}$ in scenarios 1 and 2 have the same mean $(i=1,2)$. In the MAP risk model described above, state 1 of the CTMC $\underline{J}$ is the 'normal' state with relatively small claims occurring at a reasonable pace (in average), while state 2 is a more dangerous/volatile environment having, in average, larger claims that occur at a higher frequency (than state 1). In Table 1, numerical values of $\boldsymbol{V}(u ; b)$ are provided when the force of interest is $\delta=4 \%$.

From Table 1 we observe that the values of $\boldsymbol{V}(u ; 50)$ are larger in scenario 2 than in scenario 1. Given that the claim size densities were chosen with an identical mean, we expect the heavy-tail Pareto distributions in scenario 2 to have a larger probability of extremes (small and large claim size values) than the light-tail exponential distributions in scenario 1 . However, the thickness in the right-hand tail of the claim size distribution has a limited impact on $V(u ; 50)$ given that ruin would likely occur for a medium-to-large claim size and that the expected discounted dividend payments $\boldsymbol{V}(u ; 50)$ do not account for the severity of the deficit at ruin. On the other hand, a larger probability of small claim sizes has a more significant impact on $\boldsymbol{V}(u ; 50)$. Indeed, it directly translates into a larger number of scenarios in which more dividends are payable for a longer period of time. This explains why the values of $\boldsymbol{V}(u ; 50)$ are as exhibited in Table 1.

TABLE 1: Values of $\boldsymbol{V}(u ; 50)$ for different initial surplus values.

\begin{tabular}{rccllc}
\hline & \multicolumn{2}{c}{ Scenario 1 } & & \multicolumn{2}{c}{ Scenario 2 } \\
\cline { 2 - 3 } \cline { 5 - 6 } & $V_{1}(u ; 50)$ & $V_{2}(u ; 50)$ & & $V_{1}(u ; 50)$ & $V_{2}(u ; 50)$ \\
\hline 5 & 31.1941 & 15.1104 & & 31.7929 & 16.8117 \\
10 & 34.0144 & 18.0166 & & 34.6264 & 19.9311 \\
25 & 43.4963 & 26.6633 & & 44.1247 & 28.8032 \\
40 & 55.1880 & 37.0533 & & 55.8268 & 39.2807 \\
50 & 64.5067 & 45.9318 & & 65.1478 & 48.1925 \\
\hline
\end{tabular}




\subsection{Higher-order moments of the discounted dividends}

To obtain an explicit expression for the higher-order moments of $D_{u, b}$, let $M_{i}(u, s ; b)$ be the moment generating function of $D_{u, b} \mid J(0)=i$, i.e.

$$
M_{i}(u, s ; b) \equiv \mathrm{E}\left[\exp \left(s D_{u, b}\right) \mid J(0)=i\right]=1+\sum_{n=1}^{\infty} \frac{s^{n}}{n !} V_{i, n}(u ; b),
$$

where $V_{i, n}(u ; b)=\mathrm{E}\left[D_{u, b}^{n} \mid J(0)=i\right]$. For notational convenience, we also define $V_{i, 0}(u ; b)=1$.

Using Itô's formula for jump diffusion processes and by letting the drift of the resulting stochastic differential equation for $V_{i, n}(u ; b)$ be 0 , we find that

$$
\begin{aligned}
& \frac{\sigma_{i}^{2}}{2} V_{i, n}^{\prime \prime}(u ; b)+c_{i} V_{i, n}^{\prime}(u ; b)-n \delta V_{i, n}(u ; b)+\sum_{k=1}^{m} D_{i k} V_{k, n}(u ; b) \\
& +\sum_{k=1}^{m} T_{i k} \int_{0}^{u} V_{k, n}(u-x ; b) f_{i k}(x) \mathrm{d} x=0, \quad 0 \leq u \leq b .
\end{aligned}
$$

Note that (17) is of the same form as (3) with $\delta$ substituted by $n \delta$. It is clear that $V_{i, n}(0 ; b)=0$ for $i \in E$. The second set of boundary conditions is obtained via the identity

$$
\left.\frac{\partial M_{i}(u, s ; b)}{\partial u}\right|_{u=b}=s M_{i}(b, s ; b),
$$

which we shall prove via a similar heuristic argument as in Gerber and Shiu (2004b). Indeed, define

$$
\Theta_{i}(u, s ; b) \equiv \mathrm{E}\left[\vartheta\left(s D_{u, b}\right) \mid J(0)=i\right],
$$

where $\vartheta$ is a nonnegative differentiable function. For the surplus process $\left\{U_{b}(t), t \geq 0\right\}$, we consider the following two situations: (a) situation 1 where the initial surplus is $b$ and (b) situation 2 where the initial surplus is $b-h$. For a 'small' and positive $h$, it is almost certain that, in both situations, the surplus will sit at the barrier level shortly (and before ruin). Under situation 1 , a total dividend of $h$ would have been paid by then. Now being at the barrier level $b$, both processes evolve identically going forward. Thus, the approximation $D_{b, b} \approx h+D_{b-h, b}$ holds, which implies that

$$
\vartheta\left(s D_{b, b}\right)-\vartheta\left(s D_{b-h, b}\right) \approx \operatorname{sh} \vartheta^{\prime}\left(s D_{b, b}\right) .
$$

Taking expectation conditional on $J(0)=i$ yields

$$
\Theta_{i}(b, s ; b)-\Theta_{i}(b-h, s ; b) \approx \operatorname{sh} \mathrm{E}\left[\vartheta^{\prime}\left(s D_{b, b}\right) \mid J(0)=i\right] .
$$

Dividing (19) by $h$ and then letting $h \rightarrow 0$, we arrive at

$$
\left.\frac{\partial \Theta_{i}(u, s ; b)}{\partial u}\right|_{u=b}=s \mathrm{E}\left[\vartheta^{\prime}\left(s D_{b, b}\right) \mid J(0)=i\right] .
$$

Equation (18) is a special case of (20) with $\vartheta(x)=\mathrm{e}^{x}$. Now, equating the coefficients of $s^{n}$ on both sides of (18), we obtain the second set of boundary conditions

$$
V_{i, n}^{\prime}(b ; b)=n V_{i, n-1}(b ; b), \quad i \in E .
$$


Owing to its similarity with (3), the solution of the integro-differential equation (17) is

$$
\boldsymbol{V}_{n}(u ; b)=n \boldsymbol{v}_{n}^{B}(u)\left[\left(\boldsymbol{v}_{n}^{B}\right)^{\prime}(b)\right]^{-1} \boldsymbol{V}_{n-1}(b ; b),
$$

or, equivalently,

$$
\boldsymbol{V}_{n}(u ; b)=n ! \boldsymbol{v}_{n}^{B}(u)\left[\left(\boldsymbol{v}_{n}^{B}\right)^{\prime}(b)\right]^{-1} \boldsymbol{v}_{n-1}^{B}(b)\left[\left(\boldsymbol{v}_{n-1}^{B}\right)^{\prime}(b)\right]^{-1} \cdots \boldsymbol{v}_{1}^{B}(b)\left[\left(\boldsymbol{v}_{1}^{B}\right)^{\prime}(b)\right]^{-1} \mathbf{1},
$$

for $0 \leq u \leq b$ and $n \in \mathbb{N}^{+}$, where $V_{n}(u ; b)=\left(V_{1, n}(u ; b), \ldots, V_{m, n}(u ; b)\right)^{\top}$. Note that the matrix $\boldsymbol{v}_{n}^{B}(u)$ in (22) corresponds to the matrix $\boldsymbol{v}^{B}(u)$ of Section 2.1, where the force of interest $\delta$ is substituted by $n \delta$. Thus, we refer the reader to the end of Section 2.1 for comments relating to the calculation of $\boldsymbol{v}_{n}^{B}(u)$.

Remark 3. An equation of the form (22) for $V_{n}(u ; b)$ has also been obtained by $\mathrm{Li}$ and $\mathrm{Lu}$ (2007) and Cheung (2007) in the Markov-modulated risk model and in the Sparre Andersen model with phase-type interclaim times, respectively.

\section{The expected discounted penalty at ruin}

In this section we are interested in deriving a result similar to the dividend-penalty identity of Gerber et al. (2006) for the class of perturbed MAP risk models. Owing to the diffusion component in (1), contributions to the expected discounted penalty function shall be decomposed on the basis of the cause of ruin: oscillation or claim. For this purpose, we define the expected discounted penalty function $\phi_{i}(u ; b)$ for $u \leq b$ (see Gerber and Shiu (1998)) as

$$
\phi_{i}(u ; b)=w_{0} \phi_{i}^{d}(u ; b)+\phi_{i}^{c}(u ; b), \quad i \in E,
$$

where $w_{0}$ is the penalty at ruin if ruin is caused by oscillation,

$$
\phi_{i}^{d}(u ; b)=\mathrm{E}\left[\mathrm{e}^{-\delta T_{b}} \mathbf{1}\left(T_{b}<\infty, U_{b}\left(T_{b}\right)=0\right) \mid U_{b}(0)=u, J(0)=i\right]
$$

is the Laplace transform of the time of ruin due to oscillation, and

$$
\begin{aligned}
\phi_{i}^{c}(u ; b)= & \mathrm{E}\left[\mathrm{e}^{-\delta T_{b}} w\left(U_{b}\left(T_{b}^{-}\right),\left|U_{b}\left(T_{b}\right)\right|\right)\right. \\
& \left.\times \mathbf{1}\left(T_{b}<\infty, U_{b}\left(T_{b}\right)<0\right) \mid U_{b}(0)=u, J(0)=i\right]
\end{aligned}
$$

is the contribution to the expected discounted penalty function due to a claim causing ruin. Here $w: \mathbb{R}^{+} \times \mathbb{R}^{+} \rightarrow \mathbb{R}$ is the so-called penalty function which is assumed to satisfy some mild integrability conditions and $\mathbf{1}(A)$ is the indicator function of the event $A$.

From Itô's formula for jump diffusion processes we easily find the following integrodifferential equations for $\phi_{i}^{d}(u ; b)$ and $\phi_{i}^{c}(u ; b)$ respectively:

$$
\begin{aligned}
& \frac{\sigma_{i}^{2}}{2}\left(\phi_{i}^{d}\right)^{\prime \prime}(u ; b)+c_{i}\left(\phi_{i}^{d}\right)^{\prime}(u ; b)-\delta \phi_{i}^{d}(u ; b)+\sum_{j=1}^{m} D_{i j} \phi_{j}^{d}(u ; b) \\
& +\sum_{j=1}^{m} T_{i j} \int_{0}^{u} \phi_{j}^{d}(u-x ; b) f_{i j}(x) \mathrm{d} x=0, \quad 0 \leq u \leq b,
\end{aligned}
$$


and

$$
\begin{aligned}
& \frac{\sigma_{i}^{2}}{2}\left(\phi_{i}^{c}\right)^{\prime \prime}(u ; b)+c_{i}\left(\phi_{i}^{c}\right)^{\prime}(u ; b)-\delta \phi_{i}^{c}(u ; b)+\sum_{j=1}^{m} D_{i j} \phi_{j}^{c}(u ; b) \\
& +\sum_{j=1}^{m} T_{i j}\left(\int_{0}^{u} \phi_{j}^{c}(u-x ; b) f_{i j}(x) \mathrm{d} x+\omega_{i j}(u)\right)=0, \quad 0 \leq u \leq b,
\end{aligned}
$$

where $\omega_{i j}(u)=\int_{u}^{\infty} w(u, x-u) f_{i j}(x) \mathrm{d} x$. Note that the integro-differential equations (26) and (27) also hold in an identical MAP risk model without barrier.

Given that ruin occurs immediately a.s. when the initial surplus is 0 ,

$$
\phi_{i}^{d}(0 ; b)=1, \quad \phi_{i}^{c}(0 ; b)=0, \quad i \in E .
$$

It can also be proven that

$$
\left(\phi_{i}^{d}\right)^{\prime}(b ; b)=\left(\phi_{i}^{c}\right)^{\prime}(b ; b)=0, \quad i \in E,
$$

using an argument similar to that in Gerber et al. (2006). For the sake of completeness, this argument is given here. We consider two cases: $U_{b}(0)=b$ and $U_{b}(0)=b-h$, where $h$ is a 'small' and positive number. For the latter case, the surplus process almost certainly reaches the barrier level $b$ shortly (and before ruin). At this time, the sample paths of the two surplus processes coincide as does their penalty in the event of ruin. This completes the proof of (29).

Let

$$
\phi_{i}^{c}(u)=\mathrm{E}\left[\mathrm{e}^{-\delta T} w\left(U\left(T^{-}\right),|U(T)|\right) \mathbf{1}(T<\infty, U(T)<0) \mid U(0)=u, J(0)=i\right]
$$

be the contribution to the expected discounted penalty function due to a claim causing ruin in the identical MAP risk model without barrier. Equation (30) is a particular solution of the integro-differential equations (27). We also point out that (6) is the homogeneous version of (27) for which $\left\{\boldsymbol{v}_{\cdot, j}^{A}\right\}_{j=1}^{m}$ and $\left\{\boldsymbol{v}_{\cdot, j}^{B}\right\}_{j=1}^{m}$ are $2 m$ linearly independent solutions. From the general theory of integro-differential equations, it follows that

$$
\begin{aligned}
\phi_{i}^{c}(u ; b)= & \phi_{i}^{c}(u)+\sum_{j=1}^{m}\left(\phi_{j}^{c}(0 ; b)-\phi_{j}^{c}(0)\right) v_{i, j}^{A}(u) \\
& +\sum_{j=1}^{m}\left(\left(\phi_{j}^{c}\right)^{\prime}(0 ; b)-\left(\phi_{j}^{c}\right)^{\prime}(0)\right) v_{i, j}^{B}(u), \quad 0 \leq u \leq b .
\end{aligned}
$$

From the boundary conditions in (28) (which also hold in the identical MAP without barrier), (31) can be simplified to

$$
\phi_{i}^{c}(u ; b)=\phi_{i}^{c}(u)+\sum_{j=1}^{m}\left(\left(\phi_{j}^{c}\right)^{\prime}(0 ; b)-\left(\phi_{j}^{c}\right)^{\prime}(0)\right) v_{i, j}^{B}(u), \quad 0 \leq u \leq b .
$$

A matrix representation of (32) is given by

$$
\boldsymbol{\Phi}^{c}(u ; b)=\boldsymbol{\Phi}^{c}(u)+\boldsymbol{v}^{B}(u) \Upsilon \boldsymbol{},
$$


where $\boldsymbol{\Phi}^{c}(u ; b)=\left(\phi_{1}^{c}(u ; b), \ldots, \phi_{m}^{c}(u ; b)\right)^{\top}, \boldsymbol{\Phi}^{c}(u)=\left(\phi_{1}^{c}(u), \ldots, \phi_{m}^{c}(u)\right)^{\top}$, and $\Upsilon$ is an $m$-dimensional column vector whose $j$ th element is $\left(\phi_{j}^{c}\right)^{\prime}(0 ; b)-\left(\phi_{j}^{c}\right)^{\prime}(0)$. In Appendix A, the component $\boldsymbol{\Phi}^{c}(u)$ in a MAP risk model without barrier is discussed. To determine $\Upsilon$, we use the boundary conditions in (29), which yield

$$
\left(\boldsymbol{\Phi}^{c}\right)^{\prime}(b)+\left(\boldsymbol{v}^{B}\right)^{\prime}(b) \boldsymbol{\Upsilon}=\mathbf{0}^{\top} .
$$

Combining (33) and (34) yields

$$
\boldsymbol{\Phi}^{c}(u ; b)=\boldsymbol{\Phi}^{c}(u)-\boldsymbol{v}^{B}(u)\left[\left(\boldsymbol{v}^{B}\right)^{\prime}(b)\right]^{-1}\left(\boldsymbol{\Phi}^{c}\right)^{\prime}(b), \quad 0 \leq u \leq b .
$$

Note that a similar line of logic leads to the following representation for the Laplace transform of the time of ruin caused by oscillation:

$$
\boldsymbol{\Phi}^{d}(u ; b)=\boldsymbol{\Phi}^{d}(u)-\boldsymbol{v}^{B}(u)\left[\left(\boldsymbol{v}^{B}\right)^{\prime}(b)\right]^{-1}\left(\boldsymbol{\Phi}^{d}\right)^{\prime}(b), \quad 0 \leq u \leq b,
$$

where $\boldsymbol{\Phi}^{d}(u ; b)=\left(\phi_{1}^{d}(u ; b), \ldots, \phi_{m}^{d}(u ; b)\right)^{\top}$ and $\boldsymbol{\Phi}^{d}(u)=\left(\phi_{1}^{d}(u), \ldots, \phi_{m}^{d}(u)\right)^{\top}$. Note that $\phi_{i}^{d}(u)$ holds for the Laplace transform of the time to ruin caused by oscillation in the identical MAP risk model without barrier. We refer the reader to Appendix A for a discussion on $\boldsymbol{\Phi}^{d}(u)$.

Combining (23), (35), and (36), it follows that

$$
\boldsymbol{\Phi}(u ; b)=\boldsymbol{\Phi}(u)-\boldsymbol{v}^{B}(u)\left[\left(\boldsymbol{v}^{B}\right)^{\prime}(b)\right]^{-1} \boldsymbol{\Phi}^{\prime}(b), \quad 0 \leq u \leq b,
$$

where $\boldsymbol{\Phi}(u ; b)=\left(\phi_{1}(u ; b), \ldots, \phi_{m}(u ; b)\right)^{\top}$ and $\boldsymbol{\Phi}(u)=\left(\phi_{1}(u), \ldots, \phi_{m}(u)\right)^{\top}$ with $\phi_{i}(u)$ being the expected discounted penalty function in the identical MAP risk model without barrier.

Remark 4. For the class of skip-free upward stationary Markov surplus processes, the scalar versions of (11) and (37) hold and an identity, known as the dividend-penalty identity, was established by e.g. Gerber et al. (2006) between the expected discounted penalty function and the expected discounted dividend payments. In general, for the MAP risk models, the matrix structures of (11) and (37) do not allow to write $\boldsymbol{\Phi}(u ; b)$ in terms of $\boldsymbol{V}(u ; b)$ directly. Nevertheless, it is worth pointing out that the matrix $\boldsymbol{v}^{B}(u)\left[\left(\boldsymbol{v}^{B}\right)^{\prime}(b)\right]^{-1}$ plays a central role in the determination of both $\boldsymbol{\Phi}(u ; b)$ and $\boldsymbol{V}(u ; b)$.

\section{A barrier strategy dependent on the environmental process $J$}

In this section, a different dividend barrier strategy is applied to the perturbed MAP surplus process described in Section 1. In the spirit of Zhu and Yang (2008), we assume that the barrier level effective at a given time $t$ depends on the state of the CTMC $\underline{J}$ at time $t$. Let $b_{i}$ be the barrier level effective whenever the CTMC $\underline{J}$ is in state $i(i \in E)$. In what follows we are interested in studying the corresponding surplus process $\left\{U_{\boldsymbol{b}}(t), t \geq 0\right\}$ and some of its ruin-related quantities. Here $\boldsymbol{b}=\left(b_{1}, \ldots, b_{m}\right)$ is a (row) vector containing the set of barrier levels. We assume without loss of generality that the environmental states are such that $b_{i}<b_{j}$ for $i<j$.

The motivation for such a dividend payment strategy is the following: it is reasonable for an insurer to set the dividend barrier at a higher level in periods of stress (i.e. when the underlying Markovian environment is in a so-called 'dangerous' state with, in average, higher claim frequency and larger claim amounts). Indeed, at a higher barrier level, dividends are paid only if the surplus reaches a more secure level and, as a result, more capital is usually available to face the increased possibility of adverse claims experience. Once the company returns to a 'normal' state, excess reserves can be released and 'normal' dividend payments resume. 


\subsection{Analysis of a two-state environmental process}

4.1.1. Expected discounted dividend payments. Let $V_{i}(u ; \boldsymbol{b})$ be the expected discounted dividends for the surplus process $\left\{U_{\boldsymbol{b}}(t), t \geq 0\right\}$ with an initial surplus of $u$ and an initial state of the CTMC $J(0)=i$. We assume that, at the time of a transition of the Markovian process $\underline{J}$, the excess of the surplus level over the new barrier level (if positive) will be paid out as a lump-sum dividend, i.e. $V_{i}(u ; \boldsymbol{b})=V_{i}\left(b_{i} ; \boldsymbol{b}\right)+u-b_{i}$ for $u>b_{i}(i=1,2)$.

Using Itô's formula for jump diffusion processes, we readily obtain

$$
\begin{aligned}
& \frac{\sigma_{1}^{2}}{2} V_{1}^{\prime \prime}(u ; \boldsymbol{b})+c_{1} V_{1}^{\prime}(u ; \boldsymbol{b})-\delta V_{1}(u ; \boldsymbol{b})+D_{11} V_{1}(u ; \boldsymbol{b})+D_{12} V_{2}(u ; \boldsymbol{b}) \\
& +T_{11} \int_{0}^{u} V_{1}(u-x ; \boldsymbol{b}) f_{11}(x) \mathrm{d} x+T_{12} \int_{0}^{u} V_{2}(u-x ; \boldsymbol{b}) f_{12}(x) \mathrm{d} x=0, \quad 0 \leq u \leq b_{1},
\end{aligned}
$$

and

$$
\begin{aligned}
& \frac{\sigma_{2}^{2}}{2} V_{2}^{\prime \prime}(u ; \boldsymbol{b})+c_{2} V_{2}^{\prime}(u ; \boldsymbol{b})-\delta V_{2}(u ; \boldsymbol{b})+D_{21} V_{1}(u ; \boldsymbol{b})+D_{22} V_{2}(u ; \boldsymbol{b}) \\
& +T_{21} \int_{0}^{u} V_{1}(u-x ; \boldsymbol{b}) f_{21}(x) \mathrm{d} x+T_{22} \int_{0}^{u} V_{2}(u-x ; \boldsymbol{b}) f_{22}(x) \mathrm{d} x=0, \quad 0 \leq u \leq b_{2} .
\end{aligned}
$$

Using the linearly independent solutions $\left\{\boldsymbol{v}_{\cdot, j}^{A}(u)\right\}_{j=1}^{2}$ and $\left\{\boldsymbol{v}_{\cdot, j}^{B}(u)\right\}_{j=1}^{2}$ defined in (6), it follows that

$$
V_{i}(u ; \boldsymbol{b})=V_{1}^{\prime}(0 ; \boldsymbol{b}) v_{i, 1}^{B}(u)+V_{2}^{\prime}(0 ; \boldsymbol{b}) v_{i, 2}^{B}(u), \quad i=1,2, \text { for } 0 \leq u \leq b_{1} .
$$

It is clear that, for $i=1$, boundary condition (9) yields

$$
V_{1}^{\prime}\left(b_{1} ; \boldsymbol{b}\right)=V_{1}^{\prime}(0 ; \boldsymbol{b})\left(v_{1,1}^{B}\right)^{\prime}\left(b_{1}\right)+V_{2}^{\prime}(0 ; \boldsymbol{b})\left(v_{1,2}^{B}\right)^{\prime}\left(b_{1}\right)=1,
$$

while, for $i=2$, the quantity $V_{2}^{\prime}\left(b_{1} ; \boldsymbol{b}\right)$ has yet to be determined but is known to satisfy

$$
V_{2}^{\prime}\left(b_{1} ; \boldsymbol{b}\right)=V_{1}^{\prime}(0 ; \boldsymbol{b})\left(v_{2,1}^{B}\right)^{\prime}\left(b_{1}\right)+V_{2}^{\prime}(0 ; \boldsymbol{b})\left(v_{2,2}^{B}\right)^{\prime}\left(b_{1}\right) .
$$

Letting $\boldsymbol{V}(u ; \boldsymbol{b})=\left(V_{1}(u ; \boldsymbol{b}), V_{2}(u ; \boldsymbol{b})\right)^{\top}$, a matrix representation of (40) and (41) is given by

$$
\boldsymbol{V}^{\prime}(0 ; \boldsymbol{b})=\left[\left(\boldsymbol{v}^{B}\right)^{\prime}\left(b_{1}\right)\right]^{-1}\left(\begin{array}{c}
1 \\
V_{2}^{\prime}\left(b_{1} ; \boldsymbol{b}\right)
\end{array}\right) .
$$

Substituting (42) into (39) leads to

$$
\boldsymbol{V}(u ; \boldsymbol{b})=\boldsymbol{v}^{B}(u)\left[\left(\boldsymbol{v}^{B}\right)^{\prime}\left(b_{1}\right)\right]^{-1}\left(\begin{array}{c}
1 \\
V_{2}^{\prime}\left(b_{1} ; \boldsymbol{b}\right)
\end{array}\right), \quad 0 \leq u \leq b_{1} .
$$

Note that $V_{2}^{\prime}\left(b_{1} ; \boldsymbol{b}\right)$ is unknown, which implies that (43) alone does not fully characterize $\boldsymbol{V}(u ; \boldsymbol{b})$ for $0 \leq u \leq b_{1}$.

Now we consider (38) for an initial surplus $u \in\left(b_{1}, b_{2}\right)$. By letting $\xi(u)=V_{2}\left(u+b_{1} ; \boldsymbol{b}\right)$, it is immediate that

$$
\frac{\sigma_{2}^{2}}{2} \xi^{\prime \prime}(u)+c_{2} \xi^{\prime}(u)-\delta \xi(u)+D_{22} \xi(u)+T_{22} \int_{0}^{u} \xi(u-x) f_{22}(x) \mathrm{d} x+\alpha(u)=0
$$


for $0 \leq u \leq b_{2}-b_{1}$, where

$$
\begin{aligned}
\alpha(u)= & D_{21} V_{1}\left(u+b_{1} ; \boldsymbol{b}\right)+T_{21} \int_{0}^{u+b_{1}} V_{1}(x ; \boldsymbol{b}) f_{21}\left(u+b_{1}-x\right) \mathrm{d} x \\
& +T_{22} \int_{0}^{b_{1}} V_{2}(x ; \boldsymbol{b}) f_{22}\left(u+b_{1}-x\right) \mathrm{d} x \\
= & \left(D_{21}+T_{21} F_{21}(u)\right) V_{1}\left(b_{1} ; \boldsymbol{b}\right)+T_{21} \int_{0}^{b_{1}} V_{1}(x ; \boldsymbol{b}) f_{21}\left(u+b_{1}-x\right) \mathrm{d} x \\
& +T_{22} \int_{0}^{b_{1}} V_{2}(x ; \boldsymbol{b}) f_{22}\left(u+b_{1}-x\right) \mathrm{d} x+D_{21} u+T_{21} \int_{0}^{u}(u-x) f_{21}(x) \mathrm{d} x .
\end{aligned}
$$

To find the solution of the nonhomogeneous integro-differential equation (44), we rely on the use of one of its particular solutions, namely $\{\chi(u), u \geq 0\}$, which satisfies

$\frac{\sigma_{2}^{2}}{2} \chi^{\prime \prime}(u)+c_{2} \chi^{\prime}(u)-\delta \chi(u)+D_{22} \chi(u)+T_{22} \int_{0}^{u} \chi(u-x) f_{22}(x) \mathrm{d} x+\alpha(u)=0, \quad u \geq 0$,

with $\chi(0)=\chi^{\prime}(0)=0$. Clearly, the Laplace transform $\tilde{\chi}(s)=\int_{0}^{\infty} \mathrm{e}^{-s u} \chi(u) \mathrm{d} u$ is given by

$$
\tilde{\chi}(s)=-\frac{\tilde{\alpha}(s)}{\left(\sigma_{2}^{2} / 2\right) s^{2}+c_{2} s-\delta+D_{22}+T_{22} \widetilde{f}_{22}(s)},
$$

where $\widetilde{\alpha}(s)=\int_{0}^{\infty} \mathrm{e}^{-s u} \alpha(u) \mathrm{d} u$. Using (45), we readily find that

$$
\begin{aligned}
\tilde{\alpha}(s)= & \left(\frac{D_{21}}{s}+\frac{T_{21}}{s} \tilde{f}_{21}(s)\right) V_{1}\left(b_{1} ; \boldsymbol{b}\right)+\frac{D_{21}}{s^{2}}+\frac{T_{21}}{s^{2}} \tilde{f}_{21}(s) \\
& +T_{21}\left(V_{1}(; \boldsymbol{b}) * \mathcal{T}_{s} f_{21}\right)\left(b_{1}\right)+T_{22}\left(V_{2}(; \boldsymbol{b}) * \mathcal{T}_{s} f_{22}\right)\left(b_{1}\right),
\end{aligned}
$$

where $\mathcal{T}_{S}$ is the Dickson-Hipp operator (see, e.g. Dickson and Hipp (2001)) defined as

$$
\mathcal{T}_{s} f(y)=\int_{y}^{\infty} \mathrm{e}^{-s(x-y)} f(x) \mathrm{d} x, \quad y \geq 0,
$$

for $\operatorname{Re}(s) \geq 0$ and $f$ an integrable real-valued function on $[0, \infty)$, and ' $*$ ' is the convolution operator defined as

$$
(f * g)(y)=\int_{0}^{y} f(y-x) g(x) \mathrm{d} x=\int_{0}^{y} g(y-x) f(x) \mathrm{d} x=(g * f)(y), \quad y \geq 0,
$$

for two integrable functions $f$ and $g$ on $[0, \infty)$.

Also, the solution of the integro-differential equation (44) requires the identification of two linearly independent solutions, namely $\left\{y_{1}(u), u \geq 0\right\}$ and $\left\{y_{2}(u), u \geq 0\right\}$, of the associated homogeneous equation

$$
\frac{\sigma_{2}^{2}}{2} y^{\prime \prime}(u)+c_{2} y^{\prime}(u)-\delta y(u)+D_{22} y(u)+T_{22} \int_{0}^{u} y(u-x) f_{22}(x) \mathrm{d} x=0 .
$$

Remark 5. Note that a homogeneous integro-differential equation of the form (47) has already been studied in the context of the classical compound Poisson risk model perturbed by diffusion. The reader is referred to $\mathrm{Li}$ (2006) for more details on the form of two linearly independent solutions. 
By defining the initial conditions $y_{i}^{(j)}(0)=\mathbf{1}(i=j+1)$ for $i=1,2$ and $j=0,1$, it follows from the general theory on integro-differential equations that

$$
\xi(u) \equiv V_{2}\left(b_{1}+u ; \boldsymbol{b}\right)=\chi(u)+V_{2}\left(b_{1} ; \boldsymbol{b}\right) y_{1}(u)+V_{2}^{\prime}\left(b_{1} ; \boldsymbol{b}\right) y_{2}(u)
$$

for $0 \leq u \leq b_{2}-b_{1}$. Incorporating the boundary condition $V_{2}^{\prime}\left(b_{2} ; \boldsymbol{b}\right)=1$, we find that

$$
\left(y_{1}^{\prime}\left(b_{2}-b_{1}\right), y_{2}^{\prime}\left(b_{2}-b_{1}\right)\right)\left(\begin{array}{l}
V_{2}\left(b_{1} ; \boldsymbol{b}\right) \\
V_{2}^{\prime}\left(b_{1} ; \boldsymbol{b}\right)
\end{array}\right)=1-\chi^{\prime}\left(b_{2}-b_{1}\right) .
$$

Combining the second equation in (43) at $u=b_{1}$, namely

$$
V_{2}\left(b_{1} ; \boldsymbol{b}\right)=(0,1) \boldsymbol{v}^{B}\left(b_{1}\right)\left[\left(\boldsymbol{v}^{B}\right)^{\prime}\left(b_{1}\right)\right]^{-1}\left(\begin{array}{c}
1 \\
V_{2}^{\prime}\left(b_{1} ; \boldsymbol{b}\right)
\end{array}\right),
$$

(49) results in a system of two linear equations for the unknown quantities $V_{2}\left(b_{1} ; \boldsymbol{b}\right)$ and $V_{2}^{\prime}\left(b_{1} ; \boldsymbol{b}\right)$. Note that $\chi^{\prime}\left(b_{2}-b_{1}\right)$ (in (49)) does depend on $V_{2}^{\prime}\left(b_{1} ; \boldsymbol{b}\right)$ via its nonhomogeneous term $\alpha(u)$. The solution of the following system leads to a complete representation of $V_{1}(u ; \boldsymbol{b})$ and $V_{2}(u ; \boldsymbol{b})$ (via (43) and (48)).

4.1.2. Higher moments of the discounted dividends. Let $V_{i, n}(u ; \boldsymbol{b})$ be the $n$th moment of the discounted dividends before ruin for the surplus process $\left\{U_{\boldsymbol{b}}(t), t \geq 0\right\}$ with $U_{\boldsymbol{b}}(0)=u$ and $J(0)=i$. For $u>b_{i}$, we have

$$
V_{i, n}(u ; \boldsymbol{b})=\sum_{k=0}^{n}\left(\begin{array}{l}
n \\
k
\end{array}\right)\left(u-b_{i}\right)^{n-k} V_{i, k}\left(b_{i} ; \boldsymbol{b}\right), \quad i=1,2 .
$$

By the same arguments used to derive (43), it can be shown that

$$
\boldsymbol{V}_{n}(u ; \boldsymbol{b})=\boldsymbol{v}_{n}^{B}(u)\left[\left(\boldsymbol{v}_{n}^{B}\right)^{\prime}\left(b_{1}\right)\right]^{-1}\left(\begin{array}{c}
n V_{1, n-1}\left(b_{1} ; \boldsymbol{b}\right) \\
V_{2, n}^{\prime}\left(b_{1} ; \boldsymbol{b}\right)
\end{array}\right), \quad 0 \leq u \leq b_{1},
$$

where $\boldsymbol{V}_{n}(u ; \boldsymbol{b})=\left(V_{1, n}(u ; \boldsymbol{b}), V_{2, n}(u ; \boldsymbol{b})\right)^{\top}$.

Letting $\xi_{n}(u)=V_{2, n}\left(u+b_{1} ; \boldsymbol{b}\right)$, we also know that

$$
\frac{\sigma_{2}^{2}}{2} \xi_{n}^{\prime \prime}(u)+c_{2} \xi_{n}^{\prime}(u)-n \delta \xi_{n}(u)+D_{22} \xi_{n}(u)+T_{22} \int_{0}^{u} \xi_{n}(u-x) f_{22}(x) \mathrm{d} x+\alpha_{n}(u)=0
$$

for $0 \leq u \leq b_{2}-b_{1}$, where

$$
\begin{aligned}
\alpha_{n}(u)= & \sum_{k=0}^{n}\left(\begin{array}{l}
n \\
k
\end{array}\right)\left(D_{21} u^{n-k}+T_{21} \int_{0}^{u}(u-x)^{n-k} f_{21}(x) \mathrm{d} x\right) V_{1, k}\left(b_{1} ; \boldsymbol{b}\right) \\
& +T_{21} \int_{0}^{b_{1}} V_{1, n}(x ; \boldsymbol{b}) f_{21}\left(u+b_{1}-x\right) \mathrm{d} x+T_{22} \int_{0}^{b_{1}} V_{2, n}(x ; \boldsymbol{b}) f_{22}\left(u+b_{1}-x\right) \mathrm{d} x .
\end{aligned}
$$

It is well known that the solution of the integro-differential equation (51) satisfies

$$
\xi_{n}(u) \equiv V_{2, n}\left(u+b_{1} ; \boldsymbol{b}\right)=\chi_{n}(u)+V_{2, n}\left(b_{1} ; \boldsymbol{b}\right) y_{1, n}(u)+V_{2, n}^{\prime}\left(b_{1} ; \boldsymbol{b}\right) y_{2, n}(u)
$$


for $0 \leq u \leq b_{2}-b_{1}$, where $\left\{\chi_{n}(u), u \geq 0\right\}$ is a particular solution of (51) with $\chi_{n}(0)=$ $\chi_{n}^{\prime}(0)=0$, while $\left\{y_{1, n}(u), u \geq 0\right\}$ and $\left\{y_{2, n}(u), u \geq 0\right\}$ are two linearly independent solutions of the homogeneous version of (51) with $y_{i, n}^{(j)}(0)=\mathbf{1}(i=j+1)$ for $i=1,2$ and $j=0,1$. Given that $V_{2, n}^{\prime}\left(b_{2} ; \boldsymbol{b}\right)=n V_{2, n-1}\left(b_{2} ; \boldsymbol{b}\right)$, the representation (53) yields

$$
\chi_{n}^{\prime}\left(b_{2}-b_{1}\right)+V_{2, n}\left(b_{1} ; \boldsymbol{b}\right) y_{1, n}^{\prime}\left(b_{2}-b_{1}\right)+V_{2, n}^{\prime}\left(b_{1} ; \boldsymbol{b}\right) y_{2, n}^{\prime}\left(b_{2}-b_{1}\right)=n V_{2, n-1}\left(b_{2} ; \boldsymbol{b}\right) .
$$

The solution of the system of equations which consists of the second equation of (50) at $u=b_{1}$ and (54) together with the form of the solutions in (50) and (53) leads to a recursive expression for $\boldsymbol{V}_{n}(u ; \boldsymbol{b})$ in terms of $n$.

We point out that the Laplace transform of $\chi_{n}(u)$ is of the form (46) with $\widetilde{\alpha}(s)$ replaced by

$$
\begin{aligned}
\widetilde{\alpha}_{n}(s)= & \sum_{k=0}^{n} \frac{n !}{k !} \frac{1}{s^{n-k+1}}\left(D_{21}+T_{21} \tilde{f}_{21}(s)\right) V_{1, k}\left(b_{1} ; \boldsymbol{b}\right)+T_{21}\left(V_{1, n}(; \boldsymbol{b}) * \mathcal{T}_{s} f_{21}\right)\left(b_{1}\right) \\
& +T_{22}\left(V_{2, n}(; \boldsymbol{b}) * \mathcal{T}_{s} f_{22}\right)\left(b_{1}\right) .
\end{aligned}
$$

4.1.3. The expected discounted penalty at ruin. Let $\phi_{i}(u ; \boldsymbol{b}), \phi_{i}^{d}(u ; \boldsymbol{b})$, and $\phi_{i}^{c}(u ; \boldsymbol{b})$ be the analogues of (23), (24), and (25) for the surplus process $\left\{U_{\boldsymbol{b}}(t), t \geq 0\right\}$, respectively. Clearly, for $u>b_{i}$, we have $\phi_{i}(u ; \boldsymbol{b})=\phi_{i}\left(b_{i} ; \boldsymbol{b}\right), \phi_{i}^{d}(u ; \boldsymbol{b})=\phi_{i}^{d}\left(b_{i} ; \boldsymbol{b}\right)$, and $\phi_{i}^{c}(u ; \boldsymbol{b})=\phi_{i}^{c}\left(b_{i} ; \boldsymbol{b}\right)$, for $i=1,2$.

Relying on a similar sequence of arguments to obtain (43), we can show that

$$
\boldsymbol{\Phi}^{d}(u ; \boldsymbol{b})=\boldsymbol{\Phi}^{d}(u)+\boldsymbol{v}^{B}(u)\left[\left(\boldsymbol{v}^{B}\right)^{\prime}\left(b_{1}\right)\right]^{-1}\left(\begin{array}{c}
-\left(\phi_{1}^{d}\right)^{\prime}\left(b_{1}\right) \\
\left(\phi_{2}^{d}\right)^{\prime}\left(b_{1} ; \boldsymbol{b}\right)-\left(\phi_{2}^{d}\right)^{\prime}\left(b_{1}\right)
\end{array}\right)
$$

for $0 \leq u \leq b_{1}$, where $\boldsymbol{\Phi}^{d}(u ; \boldsymbol{b})=\left(\phi_{1}^{d}(u ; \boldsymbol{b}), \phi_{2}^{d}(u ; \boldsymbol{b})\right)^{\top}$. Note that $\left(\phi_{2}^{d}\right)^{\prime}\left(b_{1} ; \boldsymbol{b}\right)$ in (55) is unknown. Letting $\xi^{d}(u)=\phi_{2}^{d}\left(u+b_{1} ; \boldsymbol{b}\right)$, we also have

$$
\frac{\sigma_{2}^{2}}{2}\left(\xi^{d}\right)^{\prime \prime}(u)+c_{2}\left(\xi^{d}\right)^{\prime}(u)-\delta \xi^{d}(u)+D_{22} \xi^{d}(u)+T_{22} \int_{0}^{u} \xi^{d}(u-x) f_{22}(x) \mathrm{d} x+\alpha^{d}(u)=0
$$

for $0 \leq u \leq b_{2}-b_{1}$, where

$$
\begin{aligned}
\alpha^{d}(u)= & \left(D_{21}+T_{21} F_{21}(u)\right) \phi_{1}^{d}\left(b_{1} ; \boldsymbol{b}\right)+T_{21} \int_{0}^{b_{1}} \phi_{1}^{d}(x ; \boldsymbol{b}) f_{21}\left(u+b_{1}-x\right) \mathrm{d} x \\
& +T_{22} \int_{0}^{b_{1}} \phi_{2}^{d}(x ; \boldsymbol{b}) f_{22}\left(u+b_{1}-x\right) \mathrm{d} x .
\end{aligned}
$$

It is well known that the solution of the integro-differential equation (56) satisfies

$$
\begin{aligned}
\xi^{d}(u) & \equiv \phi_{2}^{d}\left(u+b_{1} ; \boldsymbol{b}\right) \\
& =\zeta^{d}(u)+\left(\phi_{2}^{d}\left(b_{1} ; \boldsymbol{b}\right)-\zeta^{d}(0)\right) y_{1}(u)+\left(\left(\phi_{2}^{d}\right)^{\prime}\left(b_{1} ; \boldsymbol{b}\right)-\left(\zeta^{d}\right)^{\prime}(0)\right) y_{2}(u)
\end{aligned}
$$

for $0 \leq u \leq b_{2}-b_{1}$, where $\left\{\zeta^{d}(u), u \geq 0\right\}$ is a particular solution of (56) with $\zeta^{d}(0)=$ $\left(\zeta^{d}\right)^{\prime}(0)=0$. Given that $\left(\phi_{2}^{d}\right)^{\prime}\left(b_{2} ; \boldsymbol{b}\right)=0$, the use of $(58)$ results in

$$
\begin{aligned}
& \left(\zeta^{d}\right)^{\prime}\left(b_{2}-b_{1}\right)+\left(\phi_{2}^{d}\left(b_{1} ; \boldsymbol{b}\right)-\zeta^{d}(0)\right) y_{1}^{\prime}\left(b_{2}-b_{1}\right)+\left(\left(\phi_{2}^{d}\right)^{\prime}\left(b_{1} ; \boldsymbol{b}\right)-\left(\zeta^{d}\right)^{\prime}(0)\right) y_{2}^{\prime}\left(b_{2}-b_{1}\right) \\
& \quad=0 .
\end{aligned}
$$


Therefore, the constants $\phi_{2}^{d}\left(b_{1} ; \boldsymbol{b}\right)$ and $\left(\phi_{2}^{d}\right)^{\prime}\left(b_{1} ; \boldsymbol{b}\right)$ can be solved from the system of equations which consists of (55) at $u=b_{1}$ and (59). A complete representation of $\boldsymbol{\Phi}^{d}(u ; \boldsymbol{b})$ can then be obtained by combining (55) and (58).

Similarly, for $\phi_{i}^{c}(u ; \boldsymbol{b})$, we find that

$$
\boldsymbol{\Phi}^{c}(u ; \boldsymbol{b})=\boldsymbol{\Phi}^{c}(u)+\boldsymbol{v}^{B}(u)\left[\left(\boldsymbol{v}^{B}\right)^{\prime}\left(b_{1}\right)\right]^{-1}\left(\begin{array}{c}
-\left(\phi_{1}^{c}\right)^{\prime}\left(b_{1}\right) \\
\left(\phi_{2}^{c}\right)^{\prime}\left(b_{1} ; \boldsymbol{b}\right)-\left(\phi_{2}^{c}\right)^{\prime}\left(b_{1}\right)
\end{array}\right), \quad 0 \leq u \leq b_{1},
$$

where $\boldsymbol{\Phi}^{c}(u ; \boldsymbol{b})=\left(\phi_{1}^{c}(u ; \boldsymbol{b}), \phi_{2}^{c}(u ; \boldsymbol{b})\right)^{\top}$. With $\xi^{c}(u)=\phi_{2}^{c}\left(u+b_{1} ; \boldsymbol{b}\right)$, it is clear that

$$
\frac{\sigma_{2}^{2}}{2}\left(\xi^{c}\right)^{\prime \prime}(u)+c_{2}\left(\xi^{c}\right)^{\prime}(u)-\delta \xi^{c}(u)+D_{22} \xi^{c}(u)+T_{22} \int_{0}^{u} \xi^{c}(u-x) f_{22}(x) \mathrm{d} x+\alpha^{c}(u)=0
$$

for $\leq u \leq b_{2}-b_{1}$, where

$$
\begin{aligned}
\alpha^{c}(u)= & \left(D_{21}+T_{21} F_{21}(u)\right) \phi_{1}^{c}\left(b_{1} ; \boldsymbol{b}\right)+T_{21} \int_{0}^{b_{1}} \phi_{1}^{c}(x ; \boldsymbol{b}) f_{21}\left(u+b_{1}-x\right) \mathrm{d} x \\
& +T_{22} \int_{0}^{b_{1}} \phi_{2}^{c}(x ; \boldsymbol{b}) f_{22}\left(u+b_{1}-x\right) \mathrm{d} x+T_{21} \omega_{21}\left(u+b_{1}\right)+T_{22} \omega_{22}\left(u+b_{1}\right) .
\end{aligned}
$$

The solution of (61) can be expressed as

$$
\begin{aligned}
\xi^{c}(u) & \equiv \phi_{2}^{c}\left(u+b_{1} ; \boldsymbol{b}\right) \\
& =\zeta^{c}(u)+\left(\phi_{2}^{c}\left(b_{1} ; \boldsymbol{b}\right)-\zeta^{c}(0)\right) y_{1}(u)+\left(\left(\phi_{2}^{c}\right)^{\prime}\left(b_{1} ; \boldsymbol{b}\right)-\left(\zeta^{c}\right)^{\prime}(0)\right) y_{2}(u),
\end{aligned}
$$

where $\left\{\zeta^{c}(u), u \geq 0\right\}$ is a particular solution of $(61)$ with $\zeta^{c}(0)=\left(\zeta^{c}\right)^{\prime}(0)=0$. Given that $\left(\phi_{2}^{c}\right)^{\prime}\left(b_{2} ; \boldsymbol{b}\right)=0$, the use of $(63)$ results in

$$
\begin{aligned}
& \left(\zeta^{c}\right)^{\prime}\left(b_{2}-b_{1}\right)+\left(\phi_{2}^{c}\left(b_{1} ; \boldsymbol{b}\right)-\zeta^{c}(0)\right) y_{1}^{\prime}\left(b_{2}-b_{1}\right)+\left(\left(\phi_{2}^{c}\right)^{\prime}\left(b_{1} ; \boldsymbol{b}\right)-\left(\zeta^{c}\right)^{\prime}(0)\right) y_{2}^{\prime}\left(b_{2}-b_{1}\right) \\
& \quad=0 .
\end{aligned}
$$

Here, again, a complete representation of $\boldsymbol{\Phi}^{c}(u ; \boldsymbol{b})$ is obtained by combining (60), (63), and (64).

Finally, we obtain the expected discounted penalty function $\phi_{i}(u ; \boldsymbol{b})$ via $\phi_{i}(u ; \boldsymbol{b})=$ $w_{0} \phi_{i}^{d}(u ; \boldsymbol{b})+\phi_{i}^{c}(u ; \boldsymbol{b})$ for $i=1,2$.

Remark 6. For the integro-differential equations (51), (56), and (61), their respective nonhomogeneous terms (52), (57), and (62) depend on $\left\{V_{i, k}(u ; \boldsymbol{b})\right\}_{k=1}^{n}, \phi_{i}^{d}(u ; \boldsymbol{b})$, and $\phi_{i}^{c}(u ; \boldsymbol{b})$, for $u \leq b_{1}$, which in turn are expressed in terms of the particular solutions $\boldsymbol{v}^{B}(u)$. From Section 2.1, it is known that when the claim size densities $f_{i j}$ all have a rational Laplace transform, (16) holds and an explicit expression for the nonhomogeneous terms (52), (57), and (62) follows. However, when at least one of the claim size densities does not have a rational Laplace transform, numerical Laplace transform inversion methods shall be used to evaluate $v_{i, j}^{B}(u)$. In such a case, the integral terms in the nonhomogeneous terms (52), (57), and (62) seem rather difficult to evaluate. Therefore, under the dividend barrier strategy of Section 4, claim size densities with rational Laplace transform should be used for calculation purposes. 


\subsection{Analysis for an arbitrary number of environmental states}

The above analysis assumes that the CTMC $J$ has only two environmental states. The consideration of a two-state environment process has been primarily due to its more simple mathematical tractability and, thus, easier presentation. However, it is clear that the technique presented in Section 4.1 can be readily extended to a CTMC with an arbitrary finite number of states. We highlight the procedure for the expected discounted dividend payments here.

Step 1. Consider surplus values in $\left(0, b_{1}\right)$. The complete set of integro-differential equations for the expected discounted dividend payments holds for this subset of initial surplus values. Find the form of the solution on $\left(0, b_{1}\right)$ and incorporate the first derivative condition, $V_{1}^{\prime}\left(b_{1} ; \boldsymbol{b}\right)=1$, into it.

Step 2. Consider surplus values in $\left(b_{i}, b_{i+1}\right)$. Only a subset of the original integro-differential equations holds for the expected discounted dividend payments for the corresponding surplus values. Find the form of the solution on $\left(b_{i}, b_{i+1}\right)$ and incorporate the first derivative condition $V_{i+1}^{\prime}\left(b_{i+1} ; \boldsymbol{b}\right)=1$.

Step 3. Repeat step 2 until all the values of $i$ in $\{1,2, \ldots, m-1\}$ have been considered, combine all the first derivative conditions and solve the resulting system of linear equations.

An application of this procedure leads to a complete representation of the expected discounted dividend payments. For the other ruin-related quantity of interest, one simply has to replace the first derivative condition at the barrier level by the appropriate one.

\section{Numerical example}

The purpose of this section is two-fold: first, we show the impact on the expected discounted dividend payments of a barrier level that depends on the state of the underlying CTMC $\underline{J}$ and second, the methodology proposed in Section 4.1 is illustrated and some intermediate quantities in the calculation of $\boldsymbol{V}(u ; \boldsymbol{b})$ are provided.

To this end, we revisit Example 1 of Section 2.1. Following the recommendation in Remark 6, we only consider scenario 1 here (where all the claim sizes are exponential). Table 2 contains the values of $\boldsymbol{V}(u ; \boldsymbol{b})$ for a force of interest $\delta=4 \%$ and different combinations of barrier levels. Note that, given the nature of state 2 , it is likely that a safer dividend policy will be adopted for state 2 (when compared to state 1), explaining why $b_{2} \geq b_{1}$ in the four scenarios considered in Table 2. In what follows, we provide some intermediate calculations for the case

TABLE 2: Values of $\boldsymbol{V}(u ; \boldsymbol{b})$ for different combinations of $\left(b_{1}, b_{2}\right)$.

\begin{tabular}{|c|c|c|c|c|c|c|c|c|}
\hline \multirow{3}{*}{$u$} & \multicolumn{8}{|c|}{$b$} \\
\hline & \multicolumn{2}{|c|}{$(50,50)$} & \multicolumn{2}{|c|}{$(50,75)$} & \multicolumn{2}{|c|}{$(50,100)$} & \multicolumn{2}{|c|}{$(50, \infty)$} \\
\hline & $V_{1}(u ; \boldsymbol{b})$ & $V_{2}(u ; \boldsymbol{b})$ & $V_{1}(u ; \boldsymbol{b})$ & $V_{2}(u ; \boldsymbol{b})$ & $V_{1}(u ; \boldsymbol{b})$ & $V_{2}(u ; \boldsymbol{b})$ & $V_{1}(u ; \boldsymbol{b})$ & $V_{2}(u ; \boldsymbol{b})$ \\
\hline 10 & 34.0144 & 18.0166 & 33.1172 & 17.1037 & 32.9160 & 16.8991 & 32.8565 & 16.8384 \\
\hline 25 & 43.4963 & 26.6633 & 42.3941 & 24.8339 & 42.1469 & 24.4237 & 42.0740 & 24.3023 \\
\hline 50 & 64.5067 & 45.9318 & 63.1780 & 39.6286 & 62.8801 & 38.2154 & 62.7919 & 37.7969 \\
\hline 75 & - & - & - & 59.5845 & - & 53.9792 & - & 52.3194 \\
\hline 100 & - & - & - & - & - & 74.0910 & - & 67.0930 \\
\hline
\end{tabular}


where $b_{1}=50$ and $b_{2}=75$ before making some observations on the values of $\boldsymbol{V}(u ; \boldsymbol{b})$ in Table 2. Note that all the figures provided are rounded to the nearest ten thousandth.

From (43) at $u=b_{1}(=50)$,

$$
V_{2}(50 ; \boldsymbol{b})=26.9723+18.9595 V_{2}^{\prime}(50 ; \boldsymbol{b}) .
$$

Also, using (48), it follows that

$$
V_{2}(50+u ; \boldsymbol{b})=\chi(u)+V_{2}(50 ; \boldsymbol{b}) y_{1}(u)+V_{2}^{\prime}(50 ; \boldsymbol{b}) y_{2}(u)
$$

for $0 \leq u \leq 25$, where

$$
\begin{aligned}
\chi(u)= & 0.6 u+35.5061+0.0181 \mathrm{e}^{-0.2 u}-0.0144 \mathrm{e}^{-150.0800 u}+7.7763 \mathrm{e}^{-0.0285 u} \\
& -43.2861 \mathrm{e}^{0.0585 u}+\left(2.3979-0.0031 \mathrm{e}^{-0.2 u}-0.0034 \mathrm{e}^{-150.0800 u}\right. \\
& \left.+4.3330 \mathrm{e}^{-0.0285 u}-6.7244 \mathrm{e}^{0.0585 u}\right) V_{2}\left(b_{1} ; \boldsymbol{b}\right), \\
y_{1}(u)= & 0.0005 \mathrm{e}^{-150.0800 u}-0.2472 \mathrm{e}^{-0.0285 u}+1.2467 \mathrm{e}^{0.0585 u},
\end{aligned}
$$

and

$$
y_{2}(u)=-0.0067 \mathrm{e}^{-150.0800 u}-0.0017 \mathrm{e}^{-0.0285 u}+0.0083 \mathrm{e}^{0.0585 u} .
$$

Using (66), the second boundary condition $V_{2}^{\prime}\left(b_{2} ; \boldsymbol{b}\right)=1$ becomes

$$
1=-\left(10.4236+1.7561 V_{2}^{\prime}(50 ; \boldsymbol{b})\right)+0.3178 V_{2}(50 ; \boldsymbol{b})+0.0021 V_{2}^{\prime}(50 ; \boldsymbol{b}) .
$$

Solving the system of equations (65) and (67), we find that $V_{2}(50 ; \boldsymbol{b})=39.6286$ and $V_{2}^{\prime}(50 ; \boldsymbol{b})=0.6675$. Therefore, we conclude that

$$
\begin{aligned}
V_{1}(u ; \boldsymbol{b})= & -27.3236 \mathrm{e}^{-600.0283 u}-0.0002 \mathrm{e}^{-150.0800 u}-0.5186 \mathrm{e}^{-0.4904 u}+0.0091 \mathrm{e}^{-0.2001 u} \\
& -0.2448 \mathrm{e}^{-0.0996 u}-0.9245 \mathrm{e}^{-0.0277 u}+29.0001 \mathrm{e}^{0.0156 u}+0.0024 \mathrm{e}^{0.0604 u}
\end{aligned}
$$

and

$$
\begin{aligned}
V_{2}(u ; \boldsymbol{b})= & 0.0001 \mathrm{e}^{-600.0283 u}-11.2420 \mathrm{e}^{-150.0800 u}+0.0023 \mathrm{e}^{-0.4904 u}-1.1602 \mathrm{e}^{-0.2001 u} \\
& -0.0359 \mathrm{e}^{-0.0996 u}-6.6932 \mathrm{e}^{-0.0277 u}+19.1557 \mathrm{e}^{0.0156 u}-0.0268 \mathrm{e}^{0.0604 u}
\end{aligned}
$$

for $0 \leq u \leq 50$, while

$$
\begin{aligned}
V_{2}(50+u ; \boldsymbol{b})= & 37.1068+0.6 u+0.0160 \mathrm{e}^{-0.2 u}+0.0000 \mathrm{e}^{-150.0800 u} \\
& +0.8711 \mathrm{e}^{-0.02849 u}+1.6347 \mathrm{e}^{0.0585 u}
\end{aligned}
$$

for $0 \leq u \leq 25$.

Finally, let us comment on the values of the expected discounted dividend payments in Table 2. We observe that, all else being equal, a higher barrier level $b_{2}$ in Table 2 seems to lead to a decrease in both $V_{1}(u, \boldsymbol{b})$ and $V_{2}(u, \boldsymbol{b})$ for a given surplus value $u$. However, we would like to remind the reader that there exists a trade-off with respect to the expected discounted dividend payments for a change in the barrier level (see, e.g. Li and $\mathrm{Lu}$ (2007) and the references therein). Indeed, a decrease in the barrier level $b_{2}$ implies that dividends will be paid more often but for a shorter period of time (given that ruin occurs earlier a.s.), and vice versa. Consequently, we should be careful in commenting on the effect of a change in the barrier level $b_{2}$ on the expected discounted dividend payments. 


\section{Appendix A}

The aim of this appendix is to give explicit expressions for the Laplace transform of the quantities $\boldsymbol{\Phi}^{d}(u)$ and $\boldsymbol{\Phi}^{c}(u)$ when $m=2$. Since all the techniques and procedures are almost identical to those given in Lu and Tsai (2007), details are omitted and only results are given here.

With $m=2, \boldsymbol{A}(s)$ is given by

$$
\boldsymbol{A}(s)=\left(\begin{array}{cc}
\frac{\sigma_{1}^{2}}{2} s^{2}+c_{1} s-\delta+D_{11}+T_{11} \tilde{f}_{11}(s) & D_{12}+T_{12} \tilde{f}_{12}(s) \\
D_{21}+T_{21} \tilde{f}_{21}(s) & \frac{\sigma_{2}^{2}}{2} s^{2}+c_{2} s-\delta+D_{22}+T_{22} \tilde{f}_{22}(s)
\end{array}\right)
$$

For $\delta>0$, an application of Rouché's theorem reveals that $\operatorname{det} \boldsymbol{A}(s)=0$ has two positive real roots which we shall denote by $\rho_{1}$ and $\rho_{2}$, and these are the only roots on the right half of the complex plane. If the positive security condition (2) is assumed then we have $\rho_{1} \rightarrow 0^{+}$as $\delta \rightarrow 0^{+}$.

Define

$$
\begin{gathered}
\tilde{\phi}_{i}^{c}(s)=\int_{0}^{\infty} \mathrm{e}^{-s u} \phi_{i}^{c}(u) \mathrm{d} u, \quad \tilde{\phi}_{i}^{d}(s)=\int_{0}^{\infty} \mathrm{e}^{-s u} \phi_{i}^{d}(u) \mathrm{d} u, \\
\text { and } \tilde{\omega}_{i j}(s)=\int_{0}^{\infty} \mathrm{e}^{-s u} \omega_{i j}(u) \mathrm{d} u,
\end{gathered}
$$

as well as the column vectors $\widetilde{\boldsymbol{\Phi}}^{c}(s)=\left(\tilde{\phi}_{1}^{c}(s), \tilde{\phi}_{2}^{c}(s)\right)^{\top}$ and $\widetilde{\boldsymbol{\Phi}}^{d}(s)=\left(\widetilde{\phi}_{1}^{d}(s), \tilde{\phi}_{2}^{d}(s)\right)^{\top}$. Analogous to Equations (4.11) and (4.12) of Lu and Tsai (2007), we arrive at

$$
\begin{gathered}
\tilde{\boldsymbol{\Phi}}^{c}(s)=\frac{\left(s-\rho_{1}\right)\left(s-\rho_{2}\right)}{\operatorname{det} \boldsymbol{A}(s)}\left\{\operatorname{adj} \boldsymbol{A}\left[s, \rho_{1}, \rho_{2}\right] \boldsymbol{B}^{c}\left(\rho_{2}\right)-\operatorname{adj} \boldsymbol{A}\left[s, \rho_{1}\right]\left(\begin{array}{l}
\sum_{j=1}^{2} T_{1 j} \tilde{\omega}_{1 j}\left[s, \rho_{2}\right] \\
\sum_{j=1}^{2} T_{2 j} \widetilde{\omega}_{1 j}\left[s, \rho_{2}\right]
\end{array}\right)\right. \\
\left.\quad-\operatorname{adj} \boldsymbol{A}\left(\rho_{1}\right)\left(\begin{array}{c}
\sum_{j=1}^{2} T_{1 j} \widetilde{\omega}_{1 j}\left[s, \rho_{1}, \rho_{2}\right] \\
\sum_{j=1}^{2} T_{2 j} \tilde{\omega}_{1 j}\left[s, \rho_{1}, \rho_{2}\right]
\end{array}\right)\right\}
\end{gathered}
$$

and

$$
\widetilde{\boldsymbol{\Phi}}^{d}(s)=\frac{\left(s-\rho_{1}\right)\left(s-\rho_{2}\right)}{\operatorname{det} \boldsymbol{A}(s)}\left\{\operatorname{adj} \boldsymbol{A}\left[s, \rho_{1}, \rho_{2}\right] \boldsymbol{B}^{d}\left(\rho_{2}\right)+\operatorname{adj} \boldsymbol{A}\left[s, \rho_{1}\right]\left(\begin{array}{c}
\frac{\sigma_{1}^{2}}{2} \\
\frac{\sigma_{2}^{2}}{2}
\end{array}\right)\right\},
$$

where

$$
\boldsymbol{B}^{c}\left(\rho_{2}\right)=\left[\operatorname{adj} \boldsymbol{A}\left[\rho_{1}, \rho_{2}\right]\right]^{-1} \operatorname{adj} \boldsymbol{A}\left(\rho_{1}\right)\left(\begin{array}{l}
\sum_{j=1}^{2} T_{1 j} \widetilde{\omega}_{1 j}\left[\rho_{1}, \rho_{2}\right] \\
\sum_{j=1}^{2} T_{2 j} \widetilde{\omega}_{1 j}\left[\rho_{1}, \rho_{2}\right]
\end{array}\right)
$$

and

$$
\boldsymbol{B}^{d}\left(\rho_{2}\right)=-\left[\operatorname{adj} \boldsymbol{A}\left[\rho_{1}, \rho_{2}\right]\right]^{-1} \operatorname{adj} \boldsymbol{A}\left(\rho_{1}\right)\left(\begin{array}{c}
\frac{\sigma_{1}^{2}}{2} \\
\frac{\sigma_{2}^{2}}{2}
\end{array}\right) .
$$


Here the first divided difference of any function $f$ with respect to two distinct numbers $x_{1}$ and $x_{2}$ is defined to be

$$
f\left[x_{1}, x_{2}\right]=\frac{f\left(x_{1}\right)-f\left(x_{2}\right)}{x_{1}-x_{2}},
$$

while the second divided difference with respect to three distinct numbers $x_{1}, x_{2}$, and $x_{3}$ is defined to be

$$
f\left[x_{1}, x_{2}, x_{3}\right]=\frac{f\left[x_{1}, x_{2}\right]-f\left[x_{1}, x_{3}\right]}{x_{2}-x_{3}} .
$$

In addition, the notion of divided difference has also been extended from scalar to matrix quantities (see, e.g. Lu and Tsai (2007)).

\section{Acknowledgements}

Support for David Landriault from grants from the Natural Sciences and Engineering Research Council of Canada is gratefully acknowledged, as is support for Eric C. K. Cheung from the Institute for Quantitative Finance and Insurance at the University of Waterloo.

\section{References}

Abate, J., Choudhury, G. L. And Whitt, W. (2000). An introduction to numerical transform inversion and its application to probability models. In Computational Probability, ed. W. K. Grassman, Kluwer, Norwell, MA, pp. 257-323.

Albrecher, H. AND Boxma, O. (2005). On the discounted penalty function in a Markov-dependent risk model. Insurance Math. Econom. 37, 650-672.

Ahn, S. And Ramaswami. V. (2006). Transient analysis of fluid models via elementary level-crossing arguments. Stoch. Models 22, 129-147.

Ahn, S., Badescu, A. L. And Ramaswami, V. (2007). Time dependent analysis of finite buffer fluid flows and risk models with a dividend barrier. Queueing Systems 55, 207-222.

Asmussen, S. (1989). Risk theory in a Markovian environment. Scand. Actuarial J. 1989, 69-100.

Asmussen, S. (1995). Stationary distributions via first passage times. In Advances in Queueing, CRC, Boca Raton, FL, pp. 79-102.

Badescu, A., Drekic, S. And Landriault, D. (2007). On the analysis of a multi-threshold Markovian risk model. Scand. Actuarial J. 2007, 248-260.

BADESCU, A. L. et al. (2005). Risk processes analyzed as fluid queues. Scand. Actuarial J. 2005, 127-141.

Cheung, E. C. K. (2007). Discussion of 'Moments of the dividend payments and related problems in a Markovmodulated risk model.' N. Amer. Actuarial J. 11, 145-148.

Dickson, D. C. M. And Hipp, C. (2001). On the time to ruin for Erlang(2) risk processes. Insurance Math. Econom. 29, 333-344.

Dufresne, D. (2001). On a general class of risk models. Austral. Actuarial J. 7, 755-791.

Gerber, H. U. ANd Shiu, E. S. W. (1998). On the time value of ruin. N. Amer. Actuarial J. 2, 48-78.

Gerber, H. U. And Shiu, E. S. W. (2004a). Optimal dividends: analysis with Brownian motion. N. Amer. Actuarial J. 8, $1-20$.

Gerber, H. U. AND Shiu, E. S. W. (2004b). Authors' reply: Optimal dividends: analysis with Brownian motion. N. Amer. Actuarial J. 8, 113-115.

Gerber, H. U., Lin, X. S. AND YANG, H. (2006). A note on the dividends-penalty identity and the optimal dividend barrier. ASTIN Bull. 36, 489-503.

Latouche, G. And Ramaswami, V. (1999). Introduction to Matrix Analytic Methods in Stochastic Modeling. American Statistical Association, Alexandria, VA.

LI, S. (2006). The distribution of the dividend payments in the compound Poisson risk model perturbed by diffusion. Scand. Actuarial J. 2006, 73-85.

LI, S. AND LU, Y. (2007). Moments of the dividend payments and related problems in a Markov-modulated risk model. N. Amer. Actuarial J. 11, 65-76.

LI, S. AND LU, Y. (2008). The decompositions of the discounted penalty functions and dividends-penalty identity in a Markov-modulated risk model. ASTIN Bull. 38, 53-71.

Lin, X. S., Willmot, G. E. AND Drekic, S. (2003). The compound Poisson risk model with a constant dividend barrier: analysis of the Gerber-Shiu discounted penalty function. Insurance Math. Econom. 33, 551-566. 
Lu, Y. AND TsaI, C. C.-L. (2007). The expected discounted penalty at ruin for a Markov-modulated risk process perturbed by diffusion. N. Amer. Actuarial J. 11, 136-152.

Neuts, M. F. (1989). Structured Stochastic Matrices of M/G/1 Type and Their Applications. Marcel Dekker, New York.

Ramaswami, V. (2006). Passage times in fluid models with application to risk processes. Methodology Comput. Appl. Prob. 8, 497-515.

ZHU, J. AND YANG, H. (2008). Ruin theory for a Markov regime-switching model under a threshold dividend strategy. Insurance Math. Econom. 42, 311-318. 